avons donnés plus haut représentent par conséquent essentiellement les moyennes de différentes recherches dans le même fromage.

\title{
RÉSUMÉ
}

Nous avons essayé, en nous basant sur nos connaissances actuelles de l'état de la gelée du fromage, de caractériser d'une façon assez précise la masse du fromage et de montrer également dans quelle direction la pratique de la fabrication des fromages doit attendre des éclaircissements nouveaux et plus complets des recherches de la chimie colloïdale.

Nous proposons comme méthodes de recherches physiques pour le fromage d'Emmental :

$1^{\circ}$ De rechercher le degré de dureté et respectivement de résistance à la pénétration ;

$2^{\circ}$ De déterminer l'élasticité, savoir :

a) en éprouvant le fromage au point de vue de la pression,

b) en l'essayant au point de vue de la traction ;

$3^{\circ}$ De déterminer la résistance à la déchirure.

En nous servant de quelques exemples de nos analyses, nous avons montré le parti que l'on pouvait tirer dans ce but des méthodes ci-dessus désignées.

\section{L'INFECTION LATENTE DE LA MAMELLE ET SES RÉVEILS. LES MOYENS DE LA DÉPISTER}

\author{
par CH. PORCHER
}

Docteur ès Sciences physiques.

(Suite)

Dans les cas les plus simples, peut-on dire qu'elle perd son caractère spécifique? N'y a-t-il vraiment qu'une déviation du fonctionnement de la cellule spécifique mammaire permettant à celle-ci de revenir à la situation ante lors de la guérisori, vraie ou apparente ?

Peut-on admettre que l'infection, en déversant des produits irritants dans les espaces lacunaires, trouble la composition chimique du liguide qui baigne les cellules acineuses, et du même coup celle du lait sécrété ? Il est bien des degrés dans les manifestations cliniques, si variées, si diverses, depuis les invisibles jusqu'aux tapageuses, qui s'offrent à nous. N'avons-nous pas sous les yeux un exemple bien connu de large infection de la mamelle qui ne nuit nullement au fonctionnement normal de celle-ci ; aussi, devrions-nous dire plutôt large infection du lait au sein d'une mamelle dont le tissu reste apparemment indemne. C'est le type d'une maladie invisible. 
Cet exemple est celui de la culture intra-mammaire du $B$. melitensis chez la chèvre. On peut être porté à penser que l'infection mélitococcique de la mamelle, chez cette espèce domestique, soit d'origine centrale. Nous pourrions le supposer également pour le B. abortus, chez la vache. Mais, malgré le voisinage très grand des deux espèces microbiennes en question, l'origine ascendante de l'infection est peut-être plus à invoquer chez la vache que l'origine descendante. L'irrégularité de l'envahissement des quatre quartiers en serait une preuve.

Le rôle possible des associations microbiennes. - Quoi qu'il en soit, il est un fait clinique indéniable : e'est que ces deux variétés de Brucella: le Melitensis de la chèvre, l'Abortus de la vache, cultivent dans la mamelle, celle-là abondamment, celle-ci plus modestement, sans y déclancher nécessairement des manifestations inflammatoires visibles. Quelquefois, on observe des poussées de mammite perceptibles eliniquement, mais, le plus souvent, plus ou moins fugaces. Doit-on les mettre au compte des microbes susdits, ou doit-on supposer qu'elles sont les manifestations d'une association microbienne intercurrente? Chez la vache, nous voyons le $B$. abortus s'associer au streptocoque; aussi, dans le cas que nous évoquons, e'est ee dernier qui, plus que le premier, nous parait coupable.

Nous pénétrons ainsi sur un terrain nouveau également, car et nous le faisions pressentir au début de cette conférence - nous ignorons beaucoup du rôle des associations microbiennes dans la genèse et le développement des mammites.

Pour revenir à la Brucellose mammaire, on est conduit à admettre que le microbe cultive dans la glande comme il cultiverait in vitro, et que le lajt que l'on recueille n'est, après tout, qu'une culture in vitro de Brucella. Mais, encore une fois, ceci est plus vrai chez la chèvre que chez la vache, espèce dont les réactions inflammatoires mammaires sont plus marquées lors d'envahissement par l'Abortus, du fait, pensons-nous, de l'intervention d'associations microbiennes.

En tout cas, chez la chèvre, le rendement laitier n'est, pour ainsi dire, pas diminué, l'état général reste bon, et la seule chose qui soit perceptible ne l'est qu'au microscope : c'est la réaction phagocytaire. Cette réaction ne manque jamais, puisqu'il y a invasion microbienne, mais la réaction conjonctive, qui devrait suivre, est absente ou négligeable, parce que le Melitensis n'est pas un microbe dont les produits de sécrétion irritent les cellules conjonctives, d'une part, la cellule mammaire, d'autre part, au point d'en altérer le fonctionnement physiologique. L'analyse courante ne décèle rien de marqué, de bien spécial dans le lait de la chèvre qui, par sa mamelle, excrète d'une façon régulière une véritable culture de Melitensis. Il y a bien là une infection latente, mais elle est d'un type différent de celui sous lequel 
nous abritons les formes invisibles de la streptococcie mammaire bovine.

Lors de l'envahissement d'une mamelle caprine par le Brucella, var. melitensis, il y a lieu de penser, puisque, dans son ensemble, le quartier est envahi, que les localisations lobaires ou lobulaires dont nous discutions tout à l'heure dans le cas de l'infection ascendante plus particulièrement streptococcique, chez la vache, sont dues sans doute à des associations microbiennes.

LA LOCALISATION DES MAMmites. - Les considérations que nous venons de faire valoir, et qui sont relatives au mode d'infection de la mamelle, à son extension ou à sa localisation, suivant le cas, nous conduisent aux observations suivantes :

$1^{\circ}$ Quand l'infection est d'origine centrale, la contamination de la glande n'est point le fait de l'homme. Lorsque : a) le virus aphteux envahit la mamelle par la voie sanguine, $b$ ) le bacille tuberculeux vient y semer des nodules, ou, plus rarement, s'y manifester sous une forme granulique massive, $c$ ) le Melitensis chez la chèvre cultive dans le lait des acinis, comme il le ferait dans un tube à essais, l'homme n'est point directement responsable de l'une ou l'autre de ces infections.

$2^{\circ}$ Quand l'infection est ascendante, il n'en est plus de même. Elle est sans conteste le fait de l'homme; c'est la malpropreté de celui-ci qui, en conditionnant celle de l'animal et de tout ce qui l'entoure, favorise l'infection ascendante.

$3^{\circ}$ Quand l'infection est d'origine sanguine, les quatre quartiers sont frappés à la fois. Quand, au contraire, elle est d'origine externe, elle ne porte pas nécessairement sur tous les quartiers; elle se localise plutôt sur tel ou tel d'entre eux.

LES QUARTIERS POSTERIEURS ET SURTOUT DE GAUCHE SONT LE PLUS SOUVENT FRAPpés. LES RA ISONS. - Un travail très intéressant de Nоттвонм [5] montre que ce sont les quartiers postérieurs qui sont le plus souvent envahis, dans $83,33 \%$ des cas, dit-il, soit done les $5 / 6$; les quartiers antérieurs ne l'étaient que pour le dernier sixième. Examinant également s'il y avait une différence entre le quartier gauche et le quartier droit de derrière, il estime que sur les 83,33\% relevant des quartiers postérieurs, $61,11 \%$ appartiendraient au trayon gauche, $22,22 \%$ au trayon droit, et il explique cette différence de la façon suivante : Le fait que les trayons postérieurs sont plus souvent infectés que les autres tient à ce que, plus que les antérieurs, ils stagnent dans les matières excrémentitielles et aussi à ce que, au repos, ils se trouvent comprimés davantage que les trayons antérieurs entre les cuisses.

Pourquoi le quartier gauche est-il plus souvent atteint que le droit ? Cela serait dû à ce que la plupart des bovins choisissent plutôt 
le côté gauche que le droit pour se reposer, en raison de la situation de la panse à gauche. L'animal évite de se coucher sur le côté droit afin de ne pas comprimer le reste des organes digestifs avec la panse, dont le poids est considérable.

Il est curieux de noter que l'ordre de fréquence de la tuberculose mammaire, selon les quartiers, est du même ordre que pour la mammite streptococcique, alors que les modes d'infection sont dissemblables.

SVEN WALL [6], qui a étudié la tuberculose mammaire, montre aussi que l'infection de la moitié gauche de la mamelle est plus fréquente que celle de la moitié droite et que les trayons postérieurs sont beaucoup plus souvent atteints que les trayons antérieurs. Sur 115 mamelles qu'il a examinées, quartier par quartier, dans 105 cas, soit $91 \%$, la tuberculose a frappé plus particulièrement un ou deux trayons postérieurs, et cependant, dans le cas de cette maladie, il ne s'agit plus ici d'infection ascendante; la tuberculose mammaire est d'origine métastatique, voulant dire par là que les bacilles ont été apportés à la glande par le sang, en provenance d'un autre organe envahi.

Aux raisons que nous donne Nоттвонм, très plausibles dans le cas de la mammite streptococcique, et qui nous apparaissent évidemment moins probantes lorsqu'il s'agit de tuberculose, nous pourrions peut-être en ajouter une autre. Nous sommes tenté de penser que la vidange de la glande est moins complète sur le quartier postérieur, plus volumineux, et dont la traite, par suite, est plus fatigante, que sur le quartier antérieur. Les quartiers postérieurs sont done toujours et plus ou moins en état de rétention ; or, comme nous le verrons plus loin, la rétention est la circonstance favorisante principale de l'infection mammaire.

Nous devons admettre également que les associations microbiennes favorisent le développement du bacille tuberculeux, là où il est venu se fixer.

L'étude des lésions tubereuleuses de la mamelle et des laits (!) qui en proviennent, demande donc que la flore microbienne en soit complètement connue. Nous savons déjà que, souvent, on rencontre le streptocoque à côté du bacille tuberculeux, mais peut-être y a-t-il d'autres agents, plus protéolytiques sans doute, dont l'action ne peut que favoriser l'évolution du processus tuberculeux.

$$
\text { ** } *
$$

\section{LA RECHERCHE DE L'INFECTION LATENTE DE LA MAMELLE NOUS OBLIGE A EXAMINER LA GLANDE QUARTIER PAR QUARTIER.}

L'examen d'ensemble des modes d'infection de la mamelle nous 
montre que le plus ordinaire, celui qui par ses conséquences pèse le plus sur la production laitière, emprunte la voie ascendante; le streptocoque est le microbe le plus habituellement rencontré dans ce eas.

Dès l'instant que l'infection est d'origine externe, elle ne se ferá pas nécessairement par les quatre trayons à la fois; en cela, nous trouvons un élément de diagnostic fort important, sur lequel nous avons déjà appelé l'attention.

Lorsque, sans raison apparente, la sécrétion des quatre quartiers est affectée dans des proportions semblables, il faut penser à une infection générale; le plus souvent, c'est la fièvre aphteuse qui est là et l'animal a déjà de la température; ou bien, dans d'autres cas, il souffre de, troubles intestinaux graves qui ne sont pas toujours très pyrétiques. Au contraire, quand ce n'est que la production isolée d'un quartiẹr qui est atteinte, il faut penser à une mammite qui apparâit, à une infection latente qui se réveille.

La présente étude est vraiment celle des laits individuels. L'unité sur laquelle doit porter nos investigations, n'est plus la mamelle entière, mais le quartier.

POURQUOI EXAMINERA-T-ON QUARTIER PAR QUARTIER ? - NOUS serons conduit à l'entreprendre, soit directement, soit indirectement :

Directement, cela veut dire que, d'emblée, dans un but de police sanitaire, en vue de prévenir des retours offensifs de mammite grave après des poussées de fièvre aphteuse, par exemple, nous procéderons systématiquement, en temps voulu, à l'examen des mamelles de chaque individu. Sur ce point, je saurai vous indiquer tout à l'heure la conduite à suivre.

Indirectement, cela signifie que vous serez amenés à examiner les mamelles de chaque individu d'un troupeau ou d'une étable, quartier par quartier, parce que tel lait d'un ramassage restreint est devenu subitement, ou presque, impropre à la fabrication à laquelle il était ordinairement destiné. C'est un fromager qui se plaint que l'emprésurage est retardé, le caillé mou, le sérum laiteux, qu'il y a une diminution tout à la fois du rendement et de la qualité. Il faut en trouver la cause ; a priori - et l'on ne se trompe pas - elle est en dernière analyse, non pas dans la mauvaise qualité de l'ensemble du lait de la cuve, mais dans celle seulement d'une partie de ce lait, laquelle, mélangée au reste, a suffi pour altérer le tout - nous verrons plus loin de quelle manière. Il va falloir faire une sélection, examiner le lait de chaque fermier séparément, et quand on sera fixé sur le producteur dont le troupeau est responsable, on passera à l'examen des mamelles entières de chacun de ses animaux, puis à celui de chaque quartier des mamelles dont le lait total a été trouvé infecté. Ce travail est très 
facile ; il a pour lui d'être tout à la fois rapide et sûr - et nous verrons plus loin comment on y prociède (VALEncien et Panchaud [7].

Les moyens que nous mettrons en auvre sont plutôt adaptés à $7 a$ recherche des laits individuels. Toutefois, par l'exemple que nous venons de donner, nous voyons que la dilution d'un lait malade dans une grande quantité de lait normal apporte avec elle un élément de trouble, dont la présence est facilement enregistrée par un technicien attentif.

Les procédés de recherches des laits malades sont done encore valables pour des laits de mélange, mais encore ne faut-il pas que la dilution susdite soit trop considérable.

LES INCIDENTS DE FABRICATION DES PETITES ET MOYENNES exploitations. - Pour les laits de grands mélanges, ceux auxquels on doit penser dans l'approvisionnement des villes en lait de consommation courante, et qui sont pasteurisés dans des usines agencées à cet effet, les moyens de recherches seraient inopérants, et ce n'est pas en eux que l'on trouverait les meilleurs outils de contrôle. Mais pour les petites et les moyennes fabrications, il n'en est plus de même. En tout cas, le recours aux techniques sur lesquelles nous allons insister tout à l'heure, n'a encore une fois son intérêt que dans le travail de sélection qu'il entraîne.

Un diagnostic est à faire ; il est facile d'y parvenir si l'on opère convenablement. Le travail de VALENCIEN et PANCHAUD nous trace de façon parfaite la ligne de conduite qui doit être suivie pour procéder avec la plus grande rigueur à cette sélection. La technique qu'il indique est couramment utilisée depuis bien des années déjà dans le Canton de Genève en vue d'éliminer de la production les vaches à mammite. Elle n'offre aucune difficulté et, vu son efficacité, elle doit être suivie.

En raison de la grande diffusion de la mammite streptococcique, il arrivera que dans les pays à petites fruitières, on ait à certains moments de l'année des fabrications défectueuses; c'est une grosse perte en passant. On se contentera de dire que le lait est anormal, sans s'occuper d'en chercher la raison. Nous disons qu'il faut penser à l'infection, qu'il s'agit là d'un lait de mammite, et l'examen des quelques fermes qui alimentent la petite fruitière ne manquerait pas de déceler dans l'une ou l'autre d'entre elles, peut-être même dans toutes, des vaches mammiteuses. La recherche de la réaction du lait, l'épreuve du sédiment, celle de la catalase, celle du labferment, tout cela si rapidement facile à faire, en attendant d'autres épreuves plus délicates de laboratoire, nous fixerait en nous donnant raison.

\section{LA FLORE DE L'INFECTION LATENTE DE LA MAMELLE.}

Le lait n'est une sécrétion aseptique que théoriquement, et tous les 
essais que l'on a pu faire pour obtenir une quantité importante de lait absolument dépourvu de germes n'ont jamais abouti. Ce n'est même que rarement que l'on réussit à obtenir $10 \mathrm{~cm}^{3}$ de lait vraiment stérile, et encore, devons-nous faire cette réserve que les échantillons recueillis renferment peut-être des microbes qui eroissent si lentement qu'ils nous donnent l'illusion d'une stérilité qui, en réalité, n'existe pas.

\section{Les soins qu'il faut prendre pour l'échantillonnage des} laits. - La récolte du lait en vue des recherches qui sont évoquées ici doit être faite avec toutes les précautions voulues, sinon la pollution, pendant et après la traite, serait fatale et nous trouverions ainsi mélangés, dans le liquide recueilli : $a$ ) les microbes provenant de la mamelle elle-même et $b$ ) ceux que le manque de soins a laissé déverser dans le lait, e'est-à-dire ce que notre collègue W. STEcK [8], de la Faculté vétérinaire de Berne, appelle : a) les bactéries primaires, vivant dans la glande, et $b$ ) les bactéries secondaires, d'origine extramammaire. Celles-là sont des hôtes de la mamelle, celles-ci sont empruntées au milieu extérieur.

Les quartiers d'une même mamelle sont microbiologiquement indépendants l'un de l'autre. - D'une façon générale, on peut dire, en s'abritant derrière les recherches de STECK et d'autres auteurs, que les quartiers d'une même glande sont, au point de vue microbien, largement indépendants l'un de l'autre et que, pour un quartier donné, il y a une constance assez affirmée dans la qualité de su flore primaire.

Généralement, il y a toujours un mélange d'espèces microbiennes sur lesquelles une d'entre elles prédomine nettement.

Cette constance de l'envahissement microbien de certains quartiers, laquelle se dénote à un double titre : a) qualitativement, par la constatation de la même flore pendant toute la durée de l'observation, b) quantitativement, par un taux de germes assez peu variable, engage les auteurs à penser que l'infection dépasserait largement le sinus et les grands conduits galactophores pour s'enfoncer dans les plus fins de ces derniers ; il $y$ aurait comme une installation véritablement à demeure des colonies mierobiennes dans la trame parenchymateuse.

L'envahissement des quartiers durera des semaines, des mois, des années. Il pourra s'étendre sur plusieurs périodes de lactation, mais il subira à certains moments des oscillations, les unes assez faibles, les autres plus importantes, auxquelles correspondront de véritables réveils de l'infection latente antérieure. Au moment de "l'asséchement " physiologique, c'est-à-dire lorsqu'on cesse la lactation dans l'attente du prochair vêlage, nous constatons souvent de semblables réveils. Nous en relevons également dans des circonstances, cette 
fois, pathologiques, lors de l'éclosion d'épidémies aphteuses. Nous reviendrons d'ailleurs longuement sur ee point important.

$\mathrm{Au}$ début de cette conférence, nous avons fait remarquer qu'il était difficile de répondre à cette question : l'infection latente est-elle primitive ou secondaire? Nous penchions cependant vers l'hypothèse qu'elle était sans doute le plus souvent primitive et, à l'appui de cette conception, nous relevons ce fait intéressant relevé par STECK et qu'il estime devoir être fréquent en clinique : Dans une installation qui se trouvait sous son contrôle, on remarqua soudainement, dans un lait obtenu très proprement, la présence de streptocoques qui avaient jusque là manqué et qui, par la suite, demeurèrent constamment dans la glande qu'ils avaient envahie, comme par surprise.

C'est une question embarrassante que de préciser, non pas le mode d'envahissement de la glande, lequel, pour le streptocoque, se ramène toujours à une infection ascendante par le trayon; mais les circonstances qui ont pu favoriser cet envahissement. Nous nageons ici dans l'inconnu.

STECK a pu, puisqu'il suivait les animaux jour par jour, noter le changement qui s'est fait brusquement dans la flore d'une mamelle, mais, dans les conditions de la pratique, cela devient impossible. Disons-nous, encore une fois, que sur ce terrain difficile du dépistage de l'infection latente de la mamelle et de ses réveils, nous ne saisissons bien que les réveils, et encore, faut-il pour cela qu'ils aient pris une allure, sinon inquiétante, du moins assez grave pour appeler l'attention du propriétaire ou de ses employés.

$$
\text { ** * }
$$

Nous avons classifié plus haut les différents modes d'infection de la glande mammaire, mais d'une façon très générale, sans nous préoccuper de savoir s'il s'agissait d'affection aiguë ou chronique. Voyons ce qu'il faut en tirer au point de vue pratique pour l'infection latente.

L'origine sanguine des microbes que l'on trouve dans la mamelle saine n'est plus admise. La mamelle n'excrète pas les microbes que le sang convoie si elle ne présente pas de lésions. Pour la tuberculose cependant, le fait contraire a été maintes fois avancé, mais on est toujours autorisé à penser que les lésions de ramollissement extrêmement faibles qui avaient pu permettre le passage daris le lait du $B$. tuberculosis avaient échappé aux investigations du fait de leur petitesse. Rappellerons-nous les expériences de Coquot et CéSARI [9], qui injectent dans l'artère mammaire une culture du bacille tuberculeux sans recueillir un seul dans le lait provenant de cette glande?

Reste, évidemment, la question des virus filtrants. Le lait, ainsi que l'a montré LebaILLy [10], est infectant avant l'apparition de 
tout symptôme, alors que la température ne fait que commencer à monter. Il semble done bien qu'il puisse s'agir ici d'une origine sanguine.

La notion des formes filtrables de certains microbes nous apporte des suggestions nouvelles et derrière les premiers faits signalés par Rossi [11] la forme filtrable du bacille tuberculeux qu'il aurait décelée dans le lait, nous pressentons toute une série de recherches du plus haut intérêt.

La voie centripète est le plus généralement celle de l'infection latente. - Il est incontestable - tous les faits de la pratique le montrent - que c'est la voie centripète qui doit être incriminée dans l'immense majorité des cas pour expliquer l'infection latente de la mamelle. C'est elle seule qu'emprunte le microbe le plus fréquemment rencontré : le streptocoque.

Sans doute, est-ce également à elle que fait appel le $B$. abortus. Nous avions pu penser, par analogie avec ce que l'on constate chez la chèvre laitière, chez laquelle la mélitococcie peut être considérée comme une véritable septicémie, que le bacille de Bang était également d'origine sanguine. Mais l'infection de la glande par les voies externes s'explique ici très bien, étant donné que le $B$. abortus s'écoule régulièrement par les voies génitales, et que, entre celles-ci et les quartiers mammaires, surtout les postérieurs, qui sont d'abord et plus fréquemment atteints que les antérieurs, ainsi que CoolEDGE [12] l'a montré, il n'y a pas loin.

Plan de recherches à faire sur la génisse. - Il y aurait le plus grand intérêt à faire des recherches sur la flore microbienne des génisses chez lesquelles, dès la naissance, on aurait encapuchonné les trayons des mamelles en prenant soin de vérifier, de temps à autre, la parfaite étanchéité de l'obturation.

Sur le terrain expérimental, on ne met plus en doute que l'infection latente de la mamelle soit d'origine externe et qu'elle ait emprunté le canal du trayon pour s'installer.

L'infection transitoire. - Toutes les fois qu'on injecte dans une glande un microbe, même banal : prodigiosus, fluorescens, etc., on détermine - avons-nous vu - une mammite aiguë, qui disparaît assez vite. Le microbe injecté n'est pas un hôte habituel de la glande : il est rapidement phagocyté et s'il vivote dans le parenchyme, ce n'est pas pour très longtemps.

L'infection permanente. - Il n'en est plus ainsi lorsque l'infection est due à un hôte habituel de la mamelle du type des streptocoques. Ici l'espèce microbienne, nettement pathogène, est plus irritante pour le parenchyme, oì elle est habituée à vivre, où, cliniquement, elle est si souvent rencontrée. Elle se défend contre la 
phagocytose et est prête à cultiver de nouveau lorsque les circonstances sont favorables, si bien que lorsqu'une glande a été infectée par le streptocoque, on peut dire, d'accord avec tous les auteurs, qu'elle le reste, pendant fort longtemps, sinon durant toute la carrière économique de l'animal.

Notre collègue KITT, de la Faculté vétérinaire de Zurich, a pu provoquer une mammite par simple souillure de l'extrémité du trayon. L'expérience, qui n'a pas réussi dans les mains de tous les chercheurs, n'était évidemment pas très sévère. Elle était faite pour prouver que le trayeur devait être l'agent de transmission le plus habituel, ses mains, contaminées par des laits infectés, jouant ici un rôle considérable ; mais, par la répétition des traites, il y a également une répétition des ensemencements et il n'est plus surprenant que le microbe finisse par pénétrer dans la place.

LA FLORE dU PIS. - Si les espèces microbiennes que l'on rangerait, selon la classification de SтесK, dans les bactéries secondaires, c'est-àdire dans celles qui viennent polluer le lait après la récolte, sont extrêmement variées, par contre - et tous les auteurs sont d'accord. sur ce point - on est frappé par le petit nombre d'espèces appartenant à la flore primaire de STECK, à cette flore qui végète dans le tissu de la glande qu'elle a infectée et qui y reste, en dépit des traites régulièrement effectuées, pendant un temps extrêmement long, quand ce n'est pas pendant toute la vie de l'animal.

La flore de l'infection latente n'est rien autre que celle des infections visibles, plus ou moins aiguës qui s'y rattachent.

Pour être simple, nous rangerons les espèces rencontrées de la façon suivante : les microcoques, les streptocoques, les Brucella.

Les microcoques. - Ils ont des rapports étroits avec les Micrococcus pyogenes : albus et aureus, c'est-à-dire avec les staphylocoques.

Notre confrère Jonks [13], en 1918, a fait sur ce point des observations du plus grand intérêt. Il note la présence de staphylocoques dans le pis après guérison d'une mammite et constate ultérieurement leur élimination régulière, constante par le lait, au point que si on n'avait pas été au courant d'une mammite antérieure de nature staphylococcique, on aurait considéré ces coccus, de la même espèce microbienne cependant, comme appartenant à la flore normale de la mamelle, ce que nous appellerons la flore primaire de STECK.

Cette observation est suggestive. Elle nous montre comment l'on passe du pathologique au normal ou, plus exactement, à l'apparemment normal. Elle soulève aussi la question des mammites qui passent dirions-nous - inaperçues, voulant dire par là que, sans aucun doute, il y avait eu des réactions inflammatoires qu'un homme de l'art aurait relevées sans difficultés, mais qui n'avaient nullement inquiété le propriétaire. 
Les infections à microcoques sont le plus généralement bénignes. Il n'en est pas de même de celles qui sont dues aux streptocoques.

Les streptocoques. - C'est une question très embrouillée que celle des streptocoques. Ceux que l'on rencontre dans le lait en général, dans le lait courant - dirons-nous - ont deux origines :

a) les uns proviennent du pis infecté, qu'il s'agisse d'une infection vraiment latente ou d'une infection plus accentuée, cliniquement visible; b) les autres viennent du milieu extérieur : excréments, peau, instruments employés en laiterie. Ce sont des espèces différentes qu'il ne faudrait pas confondre avec les précédentes.

Plaçons-nous en face des faits, afin de procéder le plus commodément possible à la discrimination nécessaire :

Il arrive souvent lorsqu'on recueille du lait en prenant tous les soins exigibles, c'est-à-dire lorsqu'on procède aseptiquement, d'y trouver des streptocoques, alors que la mamelle est apparemment saine. Cela répond donc bien à la définition de l'infection latente.

Entre ces streptocoques et ceux que l'on trouve dans le lait courant : S. lactis aridi, etc., et qui sont d'origine externe, il y a de grandes différences dans les réactions culturales, le $p \mathrm{H}$ des cultures, la forme des grains, les dimensions de la chaîne, la pathogénịcité, etc.

D'après les recherches de nombreux auteurs, de JoNES, de VAN OIJEN, les streptocoques de la mammite s'apparenteraient plus facilement au groupe des pyogènes. Ils seraient de deux types : les hémolysants et les non-hémolysants, ceux-ci étant plus spécialement d'origine bovine, ceux-là, au contraire, d'une origine plutôt humaine, ayant un pouvoir pathogène pour l'homme que les variétés non-hémolysantes ne possèdent pas. C'est aux variétés hémolysantes que seraient dues les épidémies d'angine d'origine lactée que l'on a souvent observées aux Etats-Unis, notamment.

Nous ne nous appesantirons pas davantage sur ees questions, préférant nous placer sur le terrain de la pathologie bovine, rester dans le cadre étroit de notre sujet, sujet qui nous porte à définir exactement les relations qui doivent être établies entre les streptocoques que nous trouvons dans du lait recueilli aseptiquement de mamelles apparemment saines et ceux que nous trouvons dans des mamelles - les mêmes d'ailleurs - présentant, cette fois, des signes d'inflammation, c'est-àdire des réveils d'une infection latente antérieure, lesquels sont souvent signalés lors de l'" asséchement » de la mamelle de l'animal.

Or, ces relations sont indubitables. Tout à l'heure, avec Jones, nous avons vu comment nous passions du pathologique au normal au sujet d'une mammite aiguë à staphylocoques. Maintenant, par la constatation de réveils d'infection sur une mamelle antérieurement calme, nous établissons des relations étiologiques étroites, microbiologiquement parlant, allant du normal au pathologique. Nous avons 
passé tout à l'heure, avec le staphylocoque, de la forme aiguë à la forme latente. Cette fois, nous faisons l'inverse et nous passons avec le streptocoque de la forme latente à la forme aiguë.

Quartiers malades et quartiers apparemment sains infectés par le même streptocoque peuvent voisiner dans la même mamelle. - L'identité des streptocoques du pis normal et des streptocoques de l'asséchement, de ceux, en somme, des mammites réveillées est indubitable. Innombrables sont les faits qui plaident en faveur de cette identité. Il en est un qu'il importe de relever ici avant tout : c'est la présence, à côté de quartiers cliniquement atteints de mammite streptococcique, d'autres quartiers pourvus également de streptocoques, mais qui nous paraissent tout à fait normaux. Nous nous efforcerons de montrer tout à l'heure pourquoi tel quartier présente des phénomènes inflammatoires, alors que celui qui le touche est sain.

JoNes qui, d'une façon très judicieuse a beaucoup travaillé cette question des streptocoques de la mammite, a démontré l'identité des streptocoques isolés sur la même mamelle, les uns d'un quartier malade, les autres du quartier voisin, sain ou apparemment sain.

On peut poser en principe, dans cette question de pathologie générale, qu'un quartier envahi de streptocoques pourra toujours, à un moment ou à un autre, devenir le siège d'une mammite. Aussi, dès 1903, notre collègue JENSEN exprime-t-il l'opinion que dans tout quartier cliniqucment sain, mais logeant des streptocoques, il y a au fond une mammite streptococcique chronique, sub-chronique - effacée, dironsnous encore. Et nous en aurons la certitude en recourant aux épreuves dont nous parlerons tout à l'heure. STECK reprend la même idée en disant qu'il s'agit d'une inflammation " en miniature ".

Beaucoup de travaux ont été faits sur le streptocoque de la mammite (Zschokke [14], Staeheli [15], Veenbaas [16], Van OiJen [17], KufFerath [18], Jones [17] et, plus récemment, Carne [20]).

Le polymorphisme du streptocoque, - La lecture des documents nous montre quelques contradictions, mais elles ne touchent pas au fond de la question. Les auteurs ne sont sans doute pas d'accord. sur la longueur des chaînes, la grosseur des grains, mais, néanmoins, il semble bien qu'il s'agit toujours là d'une même espèce, dont le polymorphisme assez accentué dépend des circonstances culturales in vivo et aussi in vitro.

Dans le lait d'une même vache ou de vaches malades de la même exploitation, nous dit STAEHELI, on peut toujours, à côté de longues chaînes, en rencontrer de moyennes ou de courtes. Les formes dì chaîne courte se rencontreraient surtout dans les stades avancés, les formes à chaînes longues quand la maladie débute, immédiatement après le 
part. En réalité, dans ces dernières circonstances, le streptocoque vivait antérieurement dans le colostrum, produit de rétention par excellence, qui ressemble par sa composition au lait de mammite avancé.

Pour d'autres auteurs, au contraire, les formes longues se rencontrent dans les stades ultimes de l'affection.

L. A. Rogers et Dahlberg [21] établissent que les streptocoques de la mamelle ont une tendance à former des chaînes longues beaucoup plus marquées que les autres streptocoques. KUFFerath [22] signale les formes longues du streptocoque au débuit de l'infection; lorsque la maladie devient ehronique, elles disparaissent et l'on ne trouve plus que des formes courtes, quelquefois même de simples diplocoques.

Trommsdorff [23], d'accord avec ERnst [24], pense, au contraire, que c'est dans les premiers stades de la mammite que se reneontrent les formes diplococciques courtes qui sont trouvées dans le sédiment, devenant ensuite des formes à longue chaîne dans les derniers stades de la maladie.

Il ne semble pas, à la lecture de ces indications contradictoires, mais qui ne troublent nullement le jugement que l'on peut être amené à porter, qu'aucun auteur ait établi la moindre relation entre la forme du streptocoque, la longueur de sa chaîne, la grosseur de ses grains et la réaction du milieu.

Nous rencontrerons le streptocoque dans l'infection latente et dans des infections faibles, mais, toutefois, marquées de la mamelle, vivant dans un liquide de réaction alcaline au $p \mathrm{H}>7$ ou allant vers l'alealinité, $p \mathrm{H}<7$, mais tendant à se rapprocher de 7 .

Dans les observations qu'on pourrait être amené à faire par la suite, nous nous permettons donc d'engager les auteurs à voir si une relation peut être établie entre le $p \mathrm{H}$ et l'aspect du microbe dans le lait.

Il est un autre fait sur lequel nous voudrions appeler l'attention.

Le streptocoque de la mammite, comme le streptocoque ordinaire du lait ( $S$. lactis acidi), et ceux qui sont couramment employés dans l'industrie laitière, eultivés in vitro, développent de l'acidité. Ils fabriquent de l'acide lactique. Notre collègue VAN OIJEN [25], d'Utrecht, a même cherché à établir une distinction entre le streptocoque de la mammite bovine et les streptocoques pathogènes pour l'homme en se basant sur la valeur qu'atteint le $p H$ dans la culture artificielle $d u$ streptocoque en milieux sucrés définis. Les streptocoques pathogènes pour la vache donneraient une culture de $p \mathrm{H}=$ 4,50 , tandis que les, streptocoques pathogènes pour l'homme donneraient à leur culture un $p$ H plus élevé : 4,87 à 5,66 .

Nous ne nous arrêterons pas aujourd'hui à ces différences, et nous attirerons votre attention sur un point plus curieux. 
L'ALCALINITE dES LAITS dE MAMMITE LATENTE. - C'est un fait très important sur lequel on doit s'appuyer dans la pratique, que les laits de mammite latente - mammite "en miniature ", dirait Sтеск - et de mammites déclarées ont une réaction plutôt alcaline. Dans cette alcalinité, nous trouvons un moyen de prospection extrêmement commode, rapide et de valeur. Or, le streptocoque est un microbe acidogène. Mais lorsqu'il y a mammite, il y a altération de la composition du lait, diminution des éléments acidogènes normaux salins et substitution à eux d'éléments alcaligènes : bicarbonate et carbonate sodiques.

On peut très bien admettre que le streptocoque de la mammite fasse de l'acide in vivo comme il en fait in vitro, mais cet acide trouve assez vite de quoi être neutralisé dans l'excès d'alcali de provenance sanguine relevant de la déviation de la sécrétion. Il se fera donc du lactate de sodium qui rentrera dans la circulation générale par résorption.

Des recherches tout à fait intéressantes du point de vue scientifique sont donc à tenter ici ; elles seraient guidées par l'hyṕothèse très rationnelle que je viens d'exprimer et porteraient sur la recherche de l'acide lactique ou, plus exactement, des lactates alcalins dans le lait et la circulation de retour.

(A suivre.)

\section{BIBLIOGRAPHIE ANALYTIQUE}

\section{LES LIVRES}

\section{Semlemann (M.). - Zur Frage der Abtötung von Tuberkel-,} Typhus-, Paratyphusbazillen und Mastitis-Streptokokken in einigen heute gebräuchlichen Milcherhitzungsanlagen (Contribution à l'étude de la question de la destruction des bacilles de la tuberculose, de la typhoide et de la paratyphoïde, et des streptocoques de la mammite, dans quelques installations actuelles de chauffage de lait). Tirage à part du no 47 (pp. 765-770) de la "Deutsche Tierärtzliche Wochenschrift », 1927. M. et H. Schaper, Hanovre, éd.

Pour la destruction des bacilles de la tuberculose par la pasteurisation lente, une température de $63^{\circ}$ est suffisante, à condition qu'elle soit maintenue pendant au moins 30 minutes, ce qui donne, pour l'opération complète effectuée dans un chambreur à quatre compartiments, un minimum de 45 minutes (remplissage + chauffage + sortie). Le contrôle de la température est suffisant si chaque appareil est.muni d'un thermomètre enregistreur.

Les essais sur la résistance au chauffage des bactéries de la typhoïde et de la paratyphoïde et des streptocoques de la mammite n'ont été effectués par S. qu'au laboratoire, et ont montré que pour la destruction de la presque intégralité des 